\title{
Philosophie du langage et de la connaissance, 1995-2010
}

Jacques Bouveresse

\section{(2) OpenEdition}

Édition électronique

URL : https://journals.openedition.org/annuaire-cdf/1065

DOI : 10.4000/annuaire-cdf. 1065

ISBN : 978-2-7226-0325-7

ISSN : 2109-9227

Éditeur

Collège de France

Édition imprimée

Date de publication : 1 avril 2013

Pagination : 769-771

ISBN : 978-2-7226-0198-7

ISSN : 0069-5580

\section{Référence électronique}

Jacques Bouveresse, «Philosophie du langage et de la connaissance, 1995-2010 », L'annuaire du

Collège de France [En ligne], 112 | 2013, mis en ligne le 22 novembre 2013, consulté le 22 août 2022

URL : http://journals.openedition.org/annuaire-cdf/1065; DOI : https://doi.org/10.4000/annuaire-cdf. 1065 
Bonnefoy Y., « Varie ragioni per dipingere gli alberi », dans Lettere internazionale $\mathrm{n}^{\circ} 113$, $3^{\text {e }}$ trimestre 2012, p. 40-44, traduction en italien de Fabio Scotto.

\section{Principales autres activités}

17 octobre 2011, réception à Lausanne du Grand prix de poésie « Pierrette Micheloud ».

26 octobre, rencontre des prisonniers de Nisida à Naples

27 octobre, réception d'un doctorat honoris causa de l'Université L'Orientale à Naples et réception du Premio Napoli au Musée de Capodimonte.

17 novembre, présentation de Jacqueline Risset à l'Istituto italiano di cultura de Paris.

19 novembre, conversation avec Alain Veinstein à la Maison des écrivains.

10 janvier 2012, participation au séminaire d'Antoine Compagnon au Collège de France : «Pourquoi Baudelaire?».

25 janvier, «Poésie et archéologie », avec Jean-Paul Avice, au Colloque franco-allemand d'archéologie, à l'Université de Metz.

31 janvier, Présentation du livre Peintures murales de la France gothique à l'Université de Grenoble.

$1^{\text {er }}$ février, débat avec Daniel Lançon à l'Université de Grenoble.

18 février, «Carte blanche » à la Bibliothèque Municipale de Tours : «Écrire en l'heure présente », avec Gwenaëlle Aubry, Jean-Yves Masson et Daniel Lançon.

5 mars, réception du prix de poésie Kowalski de la Ville de Lyon à la Mairie de Lyon.

16 mars, lecture à l'École des Beaux-Arts de Tours dans la classe d'Alain Borer, avec Jacqueline Risset. - 17 mars, lecture à Chaumussay, Indre-et-Loire. - 18 mars, lecture au Château du Rivau.

2 avril, lecture et discussion avec Jérôme Thélot à la Bibliothèque de l'Arsenal.

11 mai, participation au colloque «D'autres langues que la mienne » dirigé par Michel Zink au Collège de France : «Langue, verbe, parole : les pouvoirs du parlar cantando ».

13 juin, lecture de «Raturer outre» au Lyrik Kabinett de Munich, à l'occasion de la publication de la version allemande du livre, Streichend Schreiben dans la traduction d'Elisabeth et Wolfgang Matz. Paris.

27 septembre, lecteur du «Plusieurs raisons de peindre des arbres » à la librairie Tschann,

\section{Jacques BOUVERESSE}

\section{Philosophie du langage et de la connaissance, 1995-2010}

Une part importante du travail effectué au cours de l'année 2010-2011 avait été consacrée à la préparation de l'édition électronique du texte des quatre dernières années de cours que j'ai données au Collège de France: Dans le labyrinthe: nécessité, contingence et liberté chez Leibniz (2008-2010) et Qu'est-ce qu'un système philosophique? (2006-2008). L'année 2011-2012 a été consacrée en premier lieu à l'achèvement de ce travail et le texte des cours est désormais disponible en ligne, par le site Internet du Collège de France (voir publications). La mise au point, en vue d'une édition du même type, du texte des trois années de cours que j'ai données sur 
Gödel : « Kurt Gödel : mathématiques, logique et philosophie » (2003-2004), « Kurt Gödel : mathématiques, logique et philosophie » (II) (2004-2005), «Kurt Gödel : mathématiques, logique et philosophie » (suite et fin) (2005-2006), est actuellement en cours et devrait être terminée avant la fin de l'année universitaire. À cela s'ajoutera la préparation du texte de la contribution que j'ai apportée au séminaire sur Temps, récit et fiction que j'ai dirigé pendant les années 2002-2003 et 2003-2004.

J'ai, en dehors de cela, continué à travailler à la mise au point de la version définitive de trois ouvrages, dont j'ai entrepris la rédaction il y a déjà un bon nombre d'années pour les deux premiers, et un peu plus récemment pour le troisième : Les Vagues du langage. Le "paradoxe de Wittgenstein » ou comment peut-on suivre une règle? (à paraître aux Éditions du Seuil, dans la collection Liber), la traduction française de Logical Syntax of Language de Rudolf Carnap (à paraître aux Éditions Gallimard), et Wittgenstein et Gottfried Keller, La connaissance de soi et les "difficultés de la foi » (à paraître aux Éditions Agone). Bien que le travail soit déjà très avancé dans les trois cas, je ne suis malheureusement pas encore en mesure d'indiquer avec certitude dans quel ordre et à quelle date précise ils pourront être publiés.

Au nombre des activités de cette année, je peux mentionner enfin la poursuite du travail du groupe de recherche informel sur «Philosophie et littérature », qui, par suite de contretemps et empêchements divers, n'a pu malheureusement se réunir qu'à trois reprises en 2011-2012.

\section{PuBLICATIONS}

\section{Ouvrages}

Bouveresse J., Les Lumières des positivistes, Éditions Agone, Marseille, 2011.

Bouveresse J., Qu'est-ce qu'un système philosophique ? Cours 2007 \& 2008, La philosophie de la connaissance au Collège de France, coll. "Langage et connaissance », Collège de France, 2012 ; http://philosophie-cdf.revues.org/84.

Bouveresse J., Dans le labyrinthe : nécessité, contingence et liberté chez Leibniz. Cours 2009-2010, La philosophie de la connaissance au Collège de France, coll. «Langage et connaissance », Collège de France, 2012 ; http://philosophie-cdf.revues.org/345.

Bouveresse J., À temps et à contretemps. Conférences publiques, La philosophie de la connaissance au Collège de France, coll. "Rationalité, vérité et démocratie », Collège de France, 2012 ; http://philosophie-cdf.revues.org/213.

\section{Articles}

Bouveresse $\mathrm{J}$., «Ce que des auteurs infréquentables ont à dire à ceux qui ne veulent pas leur ressembler », Revue Agone. La Philosophie malgré eux, 48, 2012, 163-185.

Bouveresse J., «Promesses et dangers de la société scientifique: les inquiétudes de Bertrand Russell », in Chevallier-le-Guyader M.-F., La Science et le débat public, Actes Sud/ IHEST, 2012, 25-80.

Bouveresse J., «Rudolf Carnap and the Legacy of Aufklärung », in Wagner P. (éd.), Carnap's Ideal of Explication and Naturalism, Palgrave Macmillan, 2012, 47-62.

Bouveresse J., «Ethique et logique : Cudworth, Leibniz, Moore et la critique du "sophisme volontariste" », contribution au volume d'hommage à Jean-Claude Pariente, sous la direction de Martine Pecharman et Philippe de Rouilhan (à paraître). 


\section{CONFÉRENCES, PARTICIPATION À DES DÉBATS}

- «La philosophie et la question de l'unité et la pluralité des savoirs», contribution au ccolloque organisé pour la célébration du vingtième anniversaire du Collège universitaire français de Moscou », 3-5 octobre 2011.

«Wittgenstein, von Wright, la science, la technique et le "mythe du progrès" », conférence donnée à la faculté de philosophie de l'université Lomonossov, Moscou, 4 octobre 2011.

«La littérature, la vérité et la connaissance », conférence donnée à l'Institut français de Saint-Pétersbourg, en réponse à une invitation de l'institut et de l'université, 10 octobre 2011.

«La place de la croyance religieuse dans un "âge séculier" », conférence donnée à l'Institut français de Madrid, 20 octobre 2012.

« Autour de Jacques Bouveresse : Satire et prophétie. Les Voix de Karl Kraus », Libreria La Central, Barcelone, avec Adan Kovacsics, Sandra Santana et Josep Canals, 26 octobre 2011.

«Science et religion», conférence donnée à l'université populaire de Gennevilliers, 14 novembre 2011.

«La valeur, la vérité et la vie», contribution au colloque organisé à l'occasion de la parution du volume I des Euvres complètes de Georges Canguilhem, université Paris I, 3 décembre 2011.

« Carnap, Cavaillès, le Mouvement de la Jeunesse et la montée du nazisme dans l'Allemagne des années trente », Conférence Cavaillès, École normale supérieure, 10 décembre 2011.

«La musique dans la famille Wittgenstein », conférence donnée au CLAS du Collège de France, 14 février 2012.

«La science et le débat public », conférence donnée dans le cadre du débat organisé par l'IHEST : «Paroles de chercheurs. Science, démocratie et débat public, Collège de France, 14 mars 2012.

«Littérature, vérité et connaissance », conférence donnée en réponse à une invitation de l'association Échange et diffusion des savoirs, Hôtel du Département, Marseille, 22 mars 2012.

«Quelques remarques sur les relations entre le principe de contradiction, le principe de perfection ou du meilleur et le principe de raison suffisante chez Leibniz », contribution au colloque organisé par Claudine Tiercelin (chaire de Métaphysique et de philosophie de la connaissance) sur «Le principe de raison chez Leibniz. Enjeux théoriques et pratiques », Collège de France, 23 mai 2012.

« Vérité, fiction et littérature », conférence donnée à l'UFR d'Arts plastiques de l'Université Paris I dans le cadre du séminaire dirigé par Jacinto Lageira, 10 octobre 2012.

«La science, la métaphysique, la religion et la question de leur avenir », conférence donnée au Colloque de rentrée du Collège de France, organisé par Henry Laurens, Hommage à Renan (11-12 octobre 2012).

«Le problème de la représentation de la réalité sociale dans le roman : quelques remarques historiques et philosophiques", contribution au colloque sur "Roman et démocratie au XIXe siècle », organisé par l'université de la Sorbonne nouvelle et l'université de Princeton, Institut finlandais, 12-13 octobre 2012.

«L'Université, la science, la démocratie et le débat public», Conférence de rentrée organisée conjointement par l'université de Lille I et l'université de Lille III, Villeneuve d'Ascq, 16 octobre 2012. 